\title{
Universiteit
}

Leiden

The Netherlands

\section{Polarization cross-coupling in a polymer microlaser upon double-pulse excitation}

Berg, S.A. van den; Sautenkov, V.A.; Hooft, G.W. 't; Eliel, E.R.

\section{Citation}

Berg, S. A. van den, Sautenkov, V. A., Hooft, G. W. 't, \& Eliel, E. R. (2002). Polarization crosscoupling in a polymer microlaser upon double-pulse excitation. Physical Review A, 65, 053821. doi:10.1103/PhysRevA.65.053821

Version: $\quad$ Not Applicable (or Unknown)

License: $\quad$ Leiden University Non-exclusive license

Downloaded from: https://hdl.handle.net/1887/61251

Note: To cite this publication please use the final published version (if applicable). 


\title{
Polarization cross-coupling in a polymer microlaser upon double-pulse excitation
}

\author{
Steven A. van den Berg,,${ }^{1, *}$ Vladimir A. Sautenkov, ${ }^{1}$ Gert W. 't Hooft, ${ }^{1,2}$ and Eric R. Eliel ${ }^{1}$ \\ ${ }^{1}$ Huygens Laboratory, Leiden University, P. O. Box 9504, 2300 RA Leiden, The Netherlands \\ ${ }^{2}$ Philips Research Laboratories, Professor Holstlaan 4, 5656 AA Eindhoven, The Netherlands
}

(Received 24 January 2002; published 9 May 2002)

\begin{abstract}
We have studied the coupling of the two polarization modes of a polymer microlaser by pumping its gain medium with two mutually delayed orthogonally polarized femtosecond pump pulses, that have a variable interpulse delay. Because the dominant anisotropy in the laser is induced by the pump and the various time scales associated with this setup are well separated, insight into the dynamics of the system is obtained by this method. Both time-resolved and time-integrated measurements of the output of the microlaser demonstrate strong cross-coupling and memory effects between the polarization modes. These can be assigned to the interplay between the optical fields in the cavity and the inversion. The most remarkable result is that the dominant output polarization switches direction when the interpulse delay is varied. Using a simple model, both for the polarization properties of the polymer and for the polymer laser, we discuss the underlying physics of the polarization cross-coupling. An attractive aspect of our gain material, i.e., a light-emitting polymer in solution, is that it has a very long memory for the polarization anisotropy induced by the pump; such a material therefore provides a transparant model system.
\end{abstract}

DOI: 10.1103/PhysRevA.65.053821

PACS number(s): 42.55.Sa, 42.65.Re, 42.70.Jk

\section{INTRODUCTION}

The polarization state of the output of a laser is a subject of recurrent interest; it has been studied for almost all types of lasers, from $\mathrm{HeNe}$ lasers in the early days [1-3] to vertical-cavity surface-emitting lasers (VCSELs) [4,5], (multimode) fiber lasers [6,7], etc. in the present day. A large part of this work has been concerned with the polarization behavior of continuous-wave (CW)-operating lasers. Recently, however, with the advent of, e.g., passively $Q$-switched single-mode microchip lasers, the pulsed regime has attracted considerable attention as well [8].

It is well known that dispersive (birefringent) and gain/ loss (dichroic) anisotropies play an important role in determining the polarization state of the light emitted by the laser; these anisotropies can have both linear and nonlinear characters. The richness of this field, and thus the recurrent interest, stems from the fact that the effective anisotropy of the laser as a whole is determined by such factors as the laser material itself, the cavity surrounding it, the pumping process, and the time scales associated with these factors. Consequently, for each new laser material or laser configuration this question has to be addressed.

Complexities arise as the various anisotropies may have different principal axes, or have different ellipticity (linear versus circular dichroism and/or birefringence). Nevertheless, most lasers can be made to oscillate in a state of stable polarization by making one anisotropy much larger than all others. For example, in a cavity with Brewster-angled interfaces the loss is so strongly anisotropic that the laser will emit linearly polarized light, even in the presence of additional anisotropies [9].

An interesting case is that where the dominant anisotropy is introduced via the pumping process; this is the case in dye

\footnotetext{
*Electronic address: sberg@molphys.leidenuniv.nl
}

lasers with isotropic cavities that are pumped by the linearly polarized output of another laser [10]. Here the anisotropy arises because the field of the pump laser selectively interacts with those dye molecules whose transition dipole is more or less aligned with the polarization vector of the pump-laser field. The effective anisotropy can be large or small, depending on the fact whether the anisotropy decays rapidly (in solvents of low viscosity), slowly (in viscous solvents) or not at all (in solid matrices) [11]. In optically pumped VCSELs a similar situation arises; a gain anisotropy can be introduced in that system by pumping with circularly polarized light. The decay of this gain anisotropy is, however, so fast [12] that it usually does not play an important role when the laser is operated in the $\mathrm{CW}$ regime. Only when the laser is driven by femtosecond pump pulses this anisotropy can be made to express itself $[13,14]$.

In recent years lasers with light-emitting polymers as gain medium have attracted considerable attention [15-18]. This new type of gain medium can be applied both as a thin solid film $[19,20]$ and in solution [21-23]. Light-emitting polymers in solution show many similarities with standard laser dyes $[16,18,21,22]$. For instance, when the polymer solution is pumped with linearly polarized light an anisotropy in the optical gain is induced, just as in a laser dye. Actually, this gain anisotropy persists for a very long time, much longer than for a laser dye that is dissolved in a solution with comparable viscosity [24]. The long memory for the pump polarization is directly related to the long reorientation time $(>1 \mathrm{~ns})$ of the extended polymer chains. Just like a dye laser the emitted polarization of the polymer laser will, therefore, be parallel to that of the pump light, if no polarizing elements are present in the cavity. Since the reorientation time exceeds the spontaneous lifetime of the polymer [24], the polymer chains can be considered fixed in space on the time scale of many experiments. A solution of light-emitting polymers, therefore, forms a perfect model system for polarization experiments. 
In the present paper we study a microcavity polymer laser by exciting the gain medium with two mutually delayed, orthogonally polarized femtosecond pulses. The double-pulse excitation leads to surprising polarization cross-coupling effects in the microlaser. These effects come to light because of the ordering of the important time scales associated with using a femtosecond pump, a microcavity laser, and a gain medium for which the relaxation of the pump-induced anisotropy is negligibly slow. By varying the delay between the two pump pulses, insight into the interplay between the two signal fields and the inversion in the microlaser is obtained.

The paper is organized as follows; in Sec. II the experimental setup is discussed, followed by the results of realtime measurements on the output of the polymer laser upon double-pulse excitation (Sec. III). A measurement of the time-integrated output in each polarization state as a function of the interpulse delay is briefly discussed. Subsequently, a model for the polymer laser is developed in which the induced anisotropies in the angular distribution of the gain are explicitly taken into account (Sec. IV). The real-time output and the effective optical gain that is associated with the individual polarization components, are calculated numerically for various values of the interpulse delay. These results provide insight into the coupling between the polarization modes in the microcavity laser. The underlying physics of the polarization cross-coupling is discussed in Sec. V. In Sec. VI the time-integrated output of both polarization components as a function of the interpulse delay is discussed more thoroughly. We show that the time-integrated data provide information on the duration of the pulse that is emitted by the microlaser. Both simulations and experimental results are presented for various pump energies, showing a strong dependence of the cross-coupling on the pump strength.

\section{EXPERIMENTAL SETUP}

To explore the polarization properties and the dynamics in the polymer microlaser, we pump its gain medium with two temporally separated, orthogonally polarized, ultrashort pulses. The microcavity consists of two flat dielectric mirrors, separated by a $17-\mu \mathrm{m}$-thin spacer. The mirrors are highly reflective for $\lambda=510-550 \mathrm{~nm}(R>99 \%)$ and nonreflective around the pump wavelength of $400 \mathrm{~nm}(T>85 \%)$. The volume between the mirrors is filled with a chlorobenzene solution (refractive index $\eta=1.523$ ) of a copolymer of two alkoxy-substituted 2-phenyl poly-( $p$-phenylenevinylene)s [25], at a concentration of $2 \mathrm{~g} / \mathrm{l}$. The polymer absorbs in the blue, while it emits in the green spectral region. The emission and absorption spectra and the repeat units are shown in Fig. 1.

The polymer laser is pumped longitudinally with 130 -fs pulses at $\lambda=400 \mathrm{~nm}$ at a repetition rate of $10 \mathrm{~Hz}$. The pump pulses are generated by a Ti:sapphire regenerative amplifier system (Spectra Physics, Spitfire), seeded by a Ti:sapphire oscillator (Kapteyn-Murnane laboratories). The 800-nm output of the Ti:sapphire laser system is frequency doubled in a 0.5 -mm-thick $\beta$-barium borate crystal. The resulting near UV beam is split into two orthogonally polarized beams by the use of a half-wave plate and a polarizing beam splitter

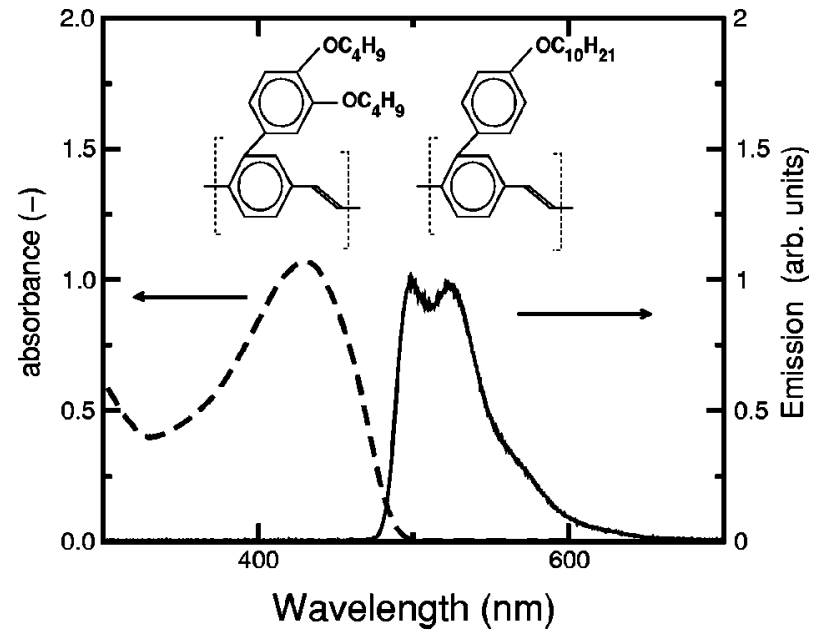

FIG. 1. Absorption (dashed line) and emission (solid line) spectra of the light-emitting copolymer dissolved in chlorobenzene. The two repeat units of the copolymer are shown above the spectra.

(see Fig. 2). The horizontally polarized pump pulse passes through a variable delay line, after which the paths of both pulses are recombined on a second polarizer. This pump beam is then brought to a focus in the laser cavity, resulting in an excitation spot with a diameter of about $10 \mu \mathrm{m}$. The two polarization components of the microlaser's output are separated by a polarizer. In part of the experiments the horizontal polarization output is recorded by a streak camera (Hamamatsu, C1587), allowing us to perform measurements in the time domain with a temporal resolution of $\approx 10 \mathrm{ps}$. In other experiments both polarization components of the output are recorded by slow photodiodes and integrated in separate gated integrators.

\section{EXPERIMENTAL RESULTS}

\section{A. Characteristics of the polymer microlaser}

First, we present some characteristics of the microlaser as it is pumped by just a single femtosecond pulse. In Fig. 3 its input-output curve is presented, displaying a clear threshold

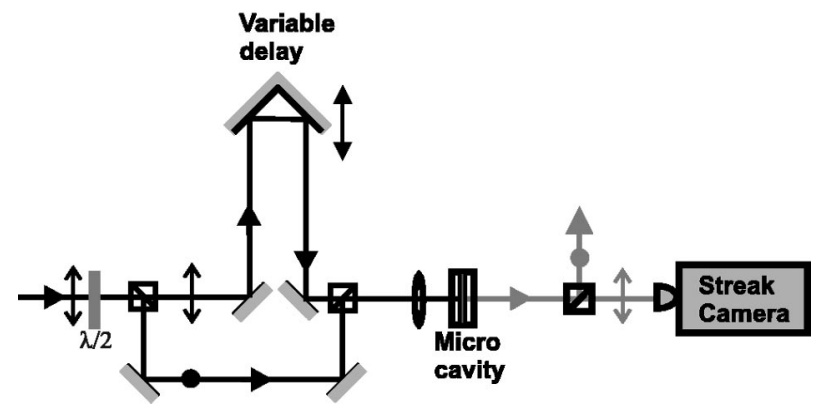

FIG. 2. Schematic of the experimental setup. A pump pulse is split into two pulses with mutually orthogonal polarizations (indicated by $\uparrow$ and $)$. The delay between the two pulses is controlled by the variable delay line. The polarization components of the light that is emitted by the microcavity laser are separated on a polarizing beam splitter and, after spectral filtering (not shown), sent to individual detectors. 


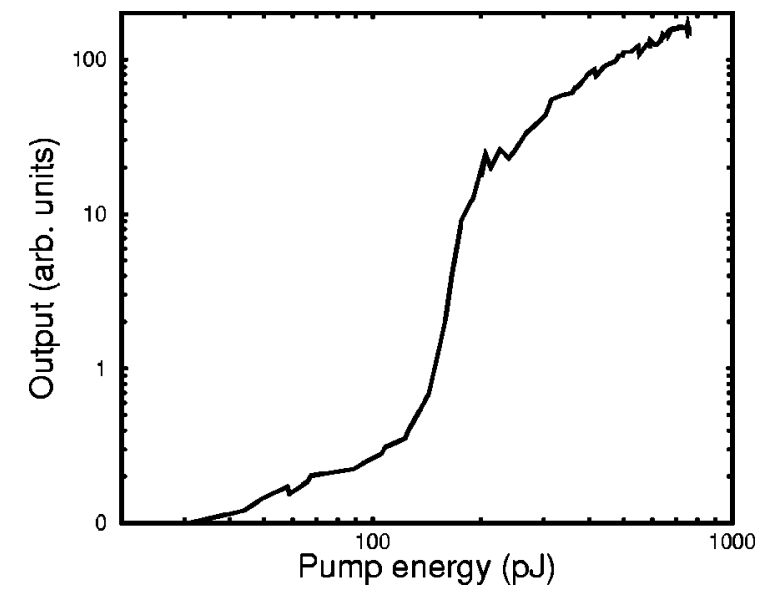

FIG. 3. Input-output curve of the polymer microlasers measured under single pulse excitation.

at a pump energy of about $0.2 \mathrm{~nJ}$. The output of the polymer laser is linearly polarized, parallel to the polarization of the pump. As expected from the cylindrical symmetry of the laser, a very similar input-output curve is obtained when the laser is pumped with the excitation beam having the orthogonal polarization.

In Fig. 4 the spectrum of the light emitted by the polymer laser is shown when the laser operates far below [Fig. 4(a)] and far above [Fig. 4(b)] threshold, respectively. The belowthreshold spectrum nicely shows the longitudinal-mode spacing, as imposed by the cavity length and the refractive index of the polymer solution. Above threshold only a few modes contribute to the laser emission of the cavity. In both spectra the width of the peaks is determined by the resolution of our spectrometer.

\section{B. Experiments in the time domain}

The results of our time-domain experiments on the output of the laser are presented in Fig. 5, for various values of the interpulse delay. The laser is pumped at about 1.6 times the threshold energy (i.e., the normalized pump parameter
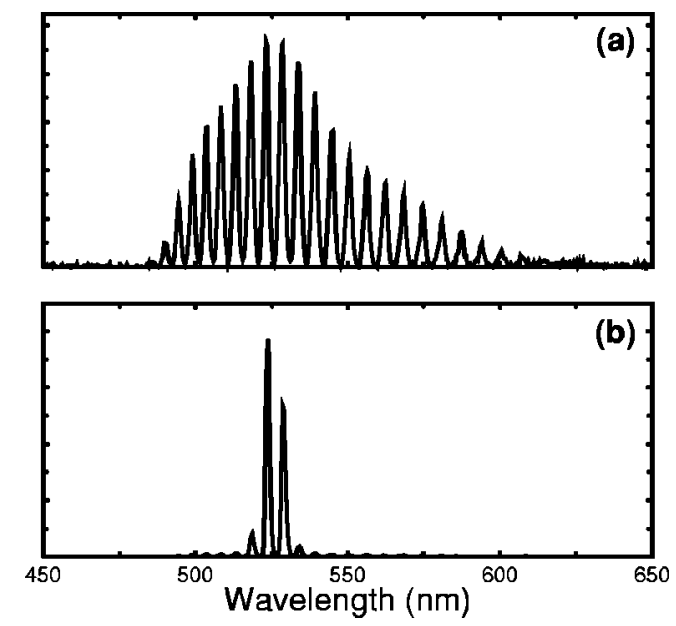

FIG. 4. Emission spectra of the polymer microlaser for excitation below (a) and far above (b) threshold, respectively.

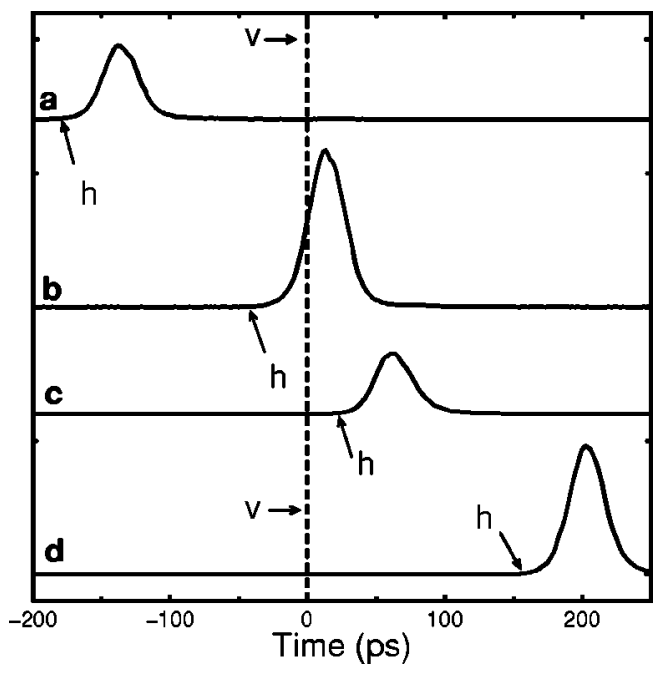

FIG. 5. Results of time-domain measurements of the horizontally polarized output of the polymer microlaser for several delay values between the horizontally and vertically polarized pumps. The vertically polarized pump pulse always arrives at $t=0$. The arrows indicate the time at which the horizontally polarized pump pulse arrives. The pump energy is approximately 1.6 times the threshold value. All curves are drawn on the same scale but have been given an offset for clarity of presentation.

$r=1.6)$. Only the horizontally polarized output component has been detected and is displayed. The experimental curves are presented in such a way that the vertically polarized pump pulse arrives at $t=0$. In curve $a$ the horizontally polarized pump pulse arrives much earlier $(\approx 180 \mathrm{ps})$ than its vertically polarized companion. The curve shows how a single pulse appears (duration $\approx 30$ ps, buildup time $\approx 40 \mathrm{ps}$ ) shortly after the horizontally polarized pump pulse, while no noticeable (horizontally polarized) output is generated after the vertically polarized pump pulse. That pump pulse generates a vertically polarized output pulse that is not detected in our setup. In short, we observe a single pulse in the horizontally polarized output channel upon excitation by two time-separated, orthogonally polarized pump pulses. It demonstrates that, for each pulse, the output polarization is completely determined by the polarization of the associated pump pulse.

The result shown in curve $a$ of Fig. 5 represents the response to the first pump pulse with the second pump pulse appearing at a time when the output of the first output pulse is completely over. It thus shows the system response to a single isolated pump pulse and, therefore, serves as a reference for further measurements.

The situation is different when the interpulse delay is short, or if the order of the pump pulses is interchanged. For instance, curve $b$ shows the output pulse for the case that the horizontal pump pulse is still first, but the interpulse delay is short (43 ps). When comparing this output pulse with that of curve $a$ one sees that the horizontally polarized output of the laser is strongly enhanced; evidently, it profits from the second (vertically polarized) pump pulse. Curve $c$ shows the situation in which the horizontally polarized pump pulse arrives somewhat after $(\approx 24 \mathrm{ps})$ the vertically polarized 


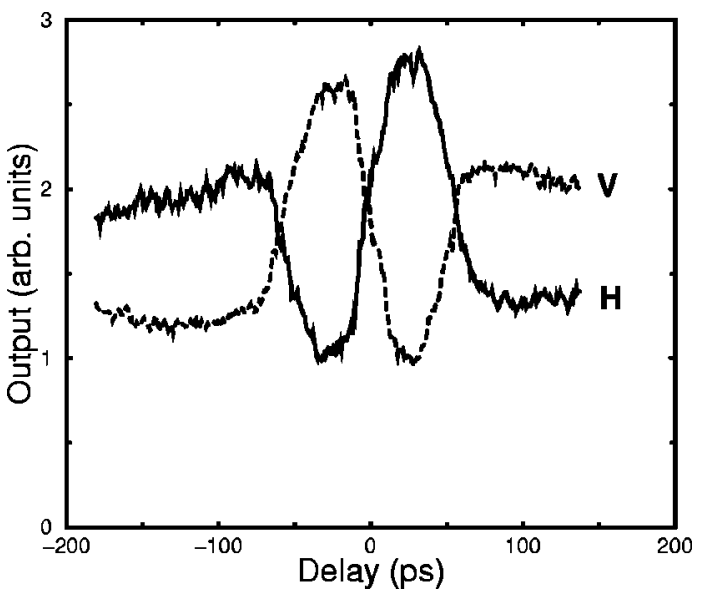

FIG. 6. Time-integrated values for the horizontally (solid line) and vertically (dashed line) polarized outputs of the polymer microlaser, measured as a function of interpulse delay. The laser is pumped approximately 1.6 times above threshold.

pump. This case appears to be unfavorable for the horizontal output; it is slightly reduced compared to the reference pulse of curve $a$. When the horizontally polarized pump pulse comes considerably later than the vertically polarized pump, as in curve $d$, the horizontally polarized output is again enhanced.

The results shown here indicate that the two polarization modes of the laser are coupled and that their coupling depends strongly on the order and delay of the two pump pulses. When comparing the various curves of Fig. 5, one notices an appreciable lack of symmetry relative to zero delay. For instance, for long interpulse delay [curves $a$ and $d$ ], the horizontally polarized output is enhanced if the last pump pulse is horizontally polarized. In contrast, for short interpulse delay as in curves $b$ and $c$, the horizontally polarized output is enhanced when the first pump pulse is horizontally polarized. This nontrivial correlation between the order of the pump pulses and the laser output suggests that memory effects are important.

\section{Time-integrated data}

In all cases discussed above we have measured the horizontally polarized output. For reasons of symmetry, the vertically polarized output shows very similar behavior. This implies that, independent of which input polarization comes first, for short interpulse delay the output polarization corresponding to the first pump pulse exceeds the output component parallel to the second pump pulse. On the contrary, for long interpulse delay the output component corresponding to the second pump pulse exceeds the output component corresponding to the first pump pulse. Consequently, at some interpulse delay, henceforth called the switch point, the output in the two polarization channels must be equal. We have explored this switch by measuring the time-integrated output for both polarizations as a function of the interpulse delay. The results are displayed in Fig. 6, showing the values of the time-integrated vertically (dashed line) and horizontally (solid line) polarized outputs of the microlaser for $r=1.6$.
For negative values of the interpulse delay the vertically polarized pump pulse excites the gain medium first, while for a positive interpulse delay the horizontal polarization comes first. Because of the symmetry around zero delay the curves are mirror images of each other with respect to $\Delta \tau=0$.

The time-integrated curves show the same overall behavior as the time-domain measurements. Indeed, one sees that the dominant output polarization of the laser switches when the interpulse delay is scanned. For this pump value the switch points lie at an interpulse delay of $\Delta \tau \approx \pm 60 \mathrm{ps}$.

\section{MODEL DESCRIPTION}

In this section we will develop a simple and effective model to describe the polarization and dynamics of the individual output pulses of our polymer laser. It is based on a combination of a specific model for the light-emitting polymer and a rate-equation description. The latter describes the evolution of the number of photons with horizontal and vertical polarizations, and that of the anisotropic inversion of the gain medium. The large difference between the duration of the pump pulse, the cavity decay time, the spontaneousemission lifetime and the dipolar-orientation relaxation time makes it possible to apply such a simple model.

\section{A. Polymer}

To describe the polarization properties of the polymer laser we view the polymer as an ensemble of dipoles that are fixed to the backbone of the polymer. Because of the random orientation of these backbones, we model our polymer solution as an ensemble of randomly oriented dipole absorbers/ emitters. For simplicity we will assume that the absorption and emission dipoles are parallel.

Absorption of linearly polarized pump light creates an anisotropic distribution of dipoles in the excited state since the probability of absorption is given by $P_{\text {abs }}=\left|\vec{\mu} \cdot \vec{E}_{\text {pump }}\right|^{2}$. Here $\vec{\mu}$ is the electric dipole moment associated with a relevant unit of the polymer, and $\vec{E}_{\text {pump }}$ represents the pump field. Our pump is sufficiently weak that we may assume that the absorption does not get saturated and that the ground state remains isotropic. One can easily show that e.g., for, a horizontally polarized pump pulse, the initial distribution of dipoles in the excited state, projected onto the polarization plane of the pump light (which is the transverse plane of the cavity in our case), is described by $N(\theta, 0)=2 N_{0} \cos ^{2} \theta$, where $N_{0}$ represents the effective number of excited dipoles. The angle $\theta$ is defined with respect to the horizontal axis.

The orientational relaxation time of our dissolved polymer has been measured to be of the order of a few nanoseconds [24], much larger than the time scale of the present experiment. We can, therefore, safely assume that the initially created anisotropy in the excited state does not relax during the experiment. In this respect the polymer in solution behaves very similar to laser dye molecules in polymer matrices [26] or rare-earth ions in amorphous hosts, such as glass [27]. The probability of emission of a linearly polarized photon into a mode of the laser cavity is given by $P_{\mathrm{em}}$ $=\left|\vec{\mu} \cdot \vec{E}_{\text {signal }}\right|^{2}=\mu^{2} E_{\text {signal }}^{2} \cos ^{2} \theta$, with $\vec{E}_{\text {signal }}$, the generated 
field. By integrating over all dipoles one can show that the intensity of the fluorescence polarized parallel to the pump polarization exceeds that of the fluorescence with orthogonal polarization by a factor of 3 [28].

\section{B. Rate equations}

In accord with many other authors $[15,16,18]$, we model our polymer laser as a homogeneously broadened, ideal fourlevel system [29]. Since the gain medium fills the cavity completely, we assume that the gains and losses are homogeneously distributed along the length of the cavity. In order to model the polarization properties of the microcavity, we take into account the angular distribution of excited dipoles, projected on the polarization plane of the pump radiation. We separately consider the horizontal and vertical polarization components of the signal field. The evolution of the inversion $N(\theta, t)$ and the horizontally and vertically polarized photon numbers $\left[n_{\mathrm{h}}(t)\right.$ and $n_{\mathrm{v}}(t)$, respectively $]$ are described by the following rate equations:

$$
\begin{gathered}
\frac{\partial N(\theta, t)}{\partial t}=-\gamma N(\theta, t)-\gamma \beta N(\theta, t)\left[n_{\mathrm{h}}(t) \cos ^{2}(\theta)\right. \\
\left.+n_{\mathrm{v}}(t) \sin ^{2}(\theta)\right], \\
\frac{d n_{\mathrm{h}}(t)}{d t}=\int_{0}^{2 \pi}\left\{\gamma \beta N(\theta, t)\left[n_{\mathrm{h}}(t)+1\right] \cos ^{2}(\theta)\right\} d \theta-\gamma_{\mathrm{c}} n_{\mathrm{h}}(t), \\
\frac{d n_{\mathrm{v}}(t)}{d t}=\int_{0}^{2 \pi}\left\{\gamma \beta N(\theta, t)\left[n_{\mathrm{v}}(t)+1\right] \sin ^{2}(\theta)\right\} d \theta-\gamma_{\mathrm{c}} n_{\mathrm{v}}(t) .
\end{gathered}
$$

Here $\gamma$ is the spontaneous-emission rate, $\gamma_{\mathrm{c}}$ the cavity decay rate, and $\beta$ denotes the fraction of spontaneous photons emitted into the lasing mode. The equations are very similar to those presented in Refs. [30] and [31]. Note that Eq. (1) does not contain the usual pump term. In our experiment the pump pulse is so short $(\approx 130 \mathrm{fs})$ compared to the other time scales that the polymer laser operates in the gainswitched regime [29]. The effect of the pump pulse is well represented by setting the value of the orientational distribution of the inversion $N(\theta, 0)$ at $t=0$. The effect of the second orthogonally polarized pump pulse is taken into account by suddenly increasing the inversion $N(\theta, \Delta \tau)$ remaining at time $\Delta \tau$ to the value $N^{\prime}(\theta, \Delta \tau)=N(\theta, \Delta \tau)+N(\theta+\pi / 2,0)$.

For the discussion below it is insightful to introduce effective gain functions for the two polarization directions:

$$
\begin{aligned}
& K(t)=\int_{0}^{2 \pi} N(\theta, t) \cos ^{2}(\theta) d \theta, \\
& M(t)=\int_{0}^{2 \pi} N(\theta, t) \sin ^{2}(\theta) d \theta .
\end{aligned}
$$

Obviously, these effective gain functions are not independent since any change of $N(\theta, t)$, will affect both $K(t)$ and $M(t)$. The rate equations then take the form

$$
\begin{aligned}
\frac{\partial N(\theta, t)}{\partial t}= & -\gamma N(\theta, t)-\gamma \beta N(\theta, t)\left[n_{\mathrm{h}}(t) \cos ^{2}(\theta)\right. \\
& \left.+n_{\mathrm{v}}(t) \sin ^{2}(\theta)\right], \\
\frac{d n_{\mathrm{h}}(t)}{d t} & =\gamma \beta\left[n_{\mathrm{h}}(t)+1\right] K(t)-\gamma_{\mathrm{c}} n_{\mathrm{h}}(t), \\
\frac{d n_{\mathrm{v}}(t)}{d t} & =\gamma \beta\left[n_{\mathrm{v}}(t)+1\right] M(t)-\gamma_{\mathrm{c}} n_{\mathrm{v}}(t) .
\end{aligned}
$$

The equations are solved numerically, using an Euler-type method in which the differentials $d t$ and $d \theta$ are approximated by finite steps.

\section{Numerical results}

We will present numerical results for the case that the first pump pulse is horizontally polarized and arrives at $t=0$. The resulting angular distribution of the inversion at $t=0$ is given by $N(\theta, 0)=2 N_{0} \cos ^{2}(\theta)$. Subsequently, this dipole distribution gets modified by spontaneous and stimulated-emission processes giving rise to an output pulse with a polarization parallel to that of the pump. At time $\Delta \tau$, the equally strong vertically polarized pump pulse arrives, adding a contribution of $2 N_{0} \sin ^{2}(\theta)$ to the inversion $N(\theta, \Delta \tau)$, which was left at that time.

In all our calculations we use the following values for the numerical coefficients: $\gamma^{-1}=0.86 \mathrm{~ns}, \gamma_{\mathrm{c}}^{-1}=2.8 \mathrm{ps}$, and $\beta$ $=4 \times 10^{-5}$. The spontaneous-emission rate $\gamma$ has been measured directly, the cavity decay rate $\gamma_{\mathrm{c}}$ is estimated from the round-trip time of the cavity and the finesse; the latter is measured to be $\mathcal{F} \approx 100$. The value for the coefficient $\beta$ is based on the cavity volume and the spectral width of the photoluminescence spectrum [32].

Figure 7(a) shows the output in both polarization modes when the interpulse delay is long (150 ps). Here the laser, as pumped by a single pump pulse, operates 1.6 times above threshold. The pump pulse that arrives at $t=0$, generates an output pulse with a buildup time of $\approx 50$ ps (peak $A_{1}$ ) and a polarization direction parallel to that of the pump. Just as curve $a$ of Fig. 5 this output pulse is the result of excitation by a single pump pulse; it, therefore, serves as a reference. The second, much delayed, orthogonally polarized pump pulse leads to a second output pulse (peak $A_{2}$ ), that is more powerful than the first pulse and is polarized parallel to its own pump pulse. Figure 7(b) shows, for this case of long interpulse delay, the evolution of the effective gain functions $K(t)$ (solid line) and $M(t)$ (dashed line), for the horizontally and vertically polarized signal fields, respectively. Note that $K(0): M(0)=3: 1$, as follows from the dipole model. Due to the second pump pulse the gain functions are boosted (with the ratio $1: 3)$ at $t=150 \mathrm{ps}$. Since some inversion is still present at that time, the gain functions reach values higher than those at $t=0$.

Figure 8(a) shows the output in both polarization modes for short interpulse delay (50 ps). In this case the optical field in the cavity, being the result of the first pump pulse, is close to its peak value when the second pump pulse arrives. Now 

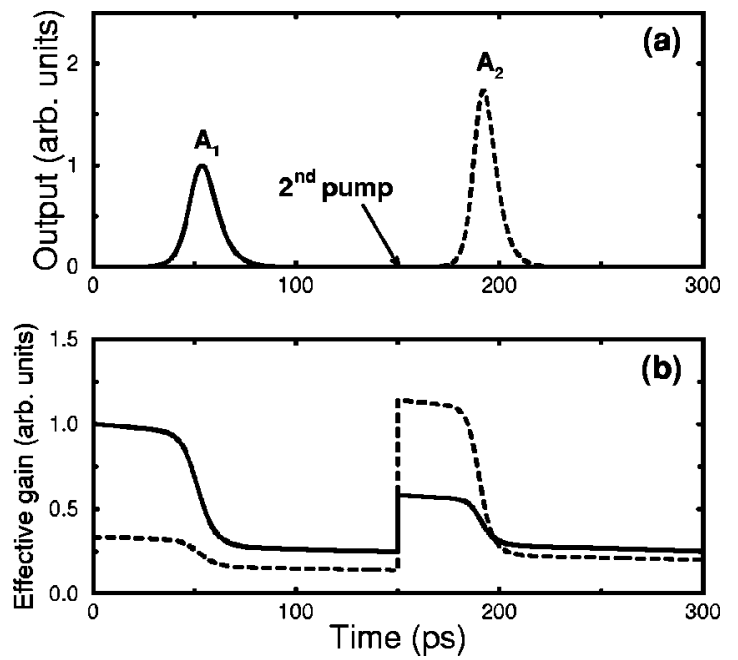

FIG. 7. Numerical simulation of the real-time horizontally (solid line) and vertically (dashed line) polarized outputs of the laser for an interpulse delay of $150 \mathrm{ps}$ (a). The horizontally polarized pump pulse arrives at $t=0$. The moment of excitation by the second, vertically polarized pulse is indicated. (b) shows the gain functions $K(t)$ (solid) and $M(t)$ (dashed) corresponding to the effective gain along the horizontal and vertical axes, respectively. The laser is pumped 1.6 times above threshold.

the first output pulse is enhanced (peak $B_{1}$ ), as compared to the reference value, while the orthogonally polarized second pulse (peak $B_{2}$ ) experiencing an increased buildup time, is suppressed. Figure 8(b) shows that in this case the gain functions are boosted just at the time when stimulated emission starts to deplete them.

In conclusion, the numerical results show, just like the experiment, the existence of two regimes of interpulse delay.
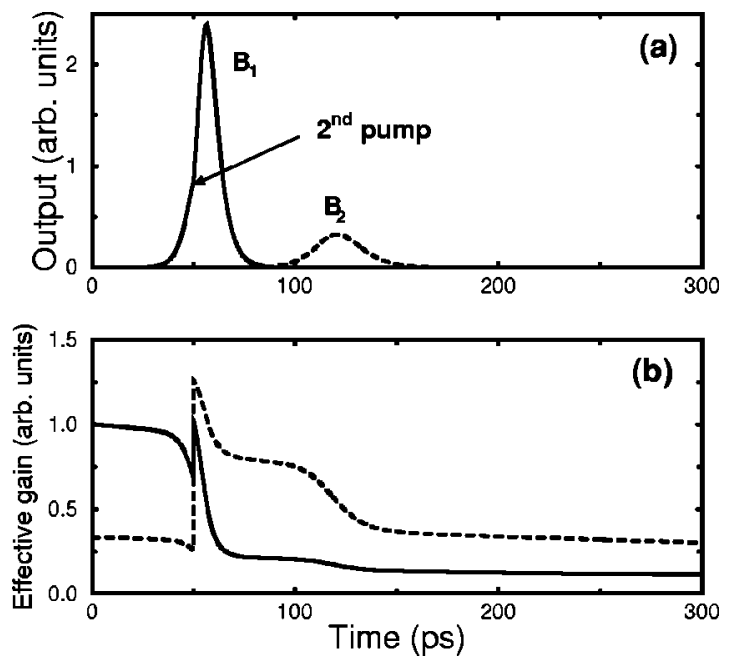

FIG. 8. Results of the numerical simulation of the horizontally (solid line) and vertically (dashed line) polarized outputs of the laser for an interpulse delay of $50 \mathrm{ps}$ (a). The horizontally polarized pump pulse arrives at $t=0$. The moment of excitation by the second, vertically polarized pulse is indicated. (b) shows the gain functions $K(t)$ (solid) and $M(t)$ (dashed), corresponding to the effective gain for the horizontal and vertical polarizations, respectively. The laser is pumped 1.6 times above threshold.
Long delays give rise to an enhanced second pulse, relative to the first pulse, while short delays generate a reduced second pulse.

\section{DISCUSSION}

The numerical results of Figs. 7 and 8 are in excellent agreement with the measurements shown in Fig. 5. Figure 7, representing the results for long interpulse delay, shows the output with polarization parallel to the first pump pulse (peak $A_{1}$ ) and that with polarization parallel to the second pump pulse (peak $A_{2}$ ). Peaks $A_{1}$ and $A_{2}$ thus correspond to curves $a$ and $d$ of Fig. 5, respectively. In the short delay regime (Fig. 8 ), peaks $B_{1}$ and $B_{2}$ represent the outputs being polarized parallel to the first and second pump pulses, respectively. Peak $B_{1}$ can be associated with curve $b$ of Fig. 5, and curve $B_{2}$ with curve $c$ of the same figure.

Having established the existence of two regimes of interpulse delay in both the experiment and the numerical calculations we will now focus on the underlying physics. When the interpulse delay is long as in Fig. 7, the first output pulse has completed its full evolution by the time the second pump pulse arrives. The power associated with the tail of the first output pulse is thus comparable with the spontaneousemission flux that is emitted by the still partially excited gain medium. The output pulse following the second pump pulse thus has to build up from the noise just as the first pulse. The increased output level of the second pulse is caused by the fact that the first pulse has not fully depleted the excitation generated by the first pump pulse [see Fig. 7(b)]. Consequently, the second output pulse can profit from this remnant. Obviously, for this picture to apply the interpulse delay should be small as compared to the spontaneous-emission lifetime. In the present experiment the time scales are such that this condition is fulfilled.

An interesting aspect of Fig. 7(b) is that it shows the coupling of the effective gain functions $K(t)$ (solid line) and $M(t)$ (dashed line) for the horizontally and vertically polarized outputs, respectively. The large initial value of $K(t)$ gives rise to a relatively fast buildup of the horizontally polarized signal field. This field depletes the inversion, affecting both gain functions $K(t)$ and $M(t)$. This coupling of the gain functions originates from the fact that any dipole in the distribution $N(\theta, t)$ contributes, with different weight factors, to both gain functions. When any dipole disappears out of the angular distribution of the inversion $N(\theta, t)$, both $K(t)$ and $M(t)$ decrease.

For short interpulse delay the evolution of the first signal pulse is not yet complete (as in Fig. 8). Now the first signal pulse can profit from the extra inversion supplied by the second pump pulse. This is well visible in Fig. 8(b) where the second pump pulse arrives at a time when the first signal pulse is effectively depleting its gain $K(t)$, represented by the solid line. Instantaneously, this gain function gets a (relatively small) boost, but now gain and signal field are large simultaneously, resulting in a big boost of the output and very efficient depletion of the gain $K(t)$. Interestingly, the gain $M(t)$ for the initially nonlasing polarization has become quite large as a result of the two pump pulses, but it is "sym- 


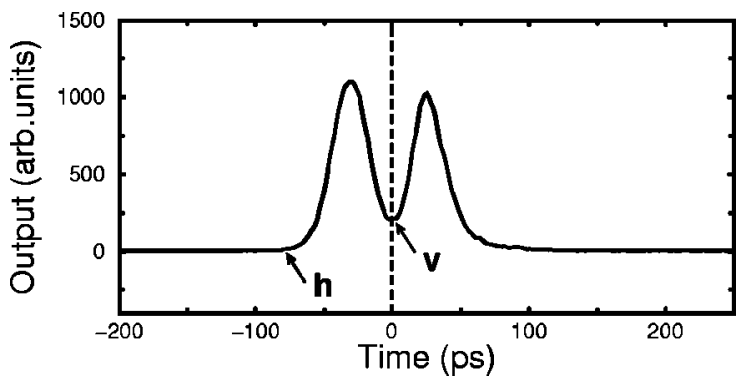

FIG. 9. Measurement of the pulse shape of the horizontally polarized output for an intermediate value of the delay $(\approx 80 \mathrm{ps})$ between the horizontally and vertically polarized pumps. The vertically polarized pump pulse arrives at $t=0$.

pathetically" depleted by the first output pulse. The remaining value of $M(t)$ is just sufficient to generate a comparatively weak vertically polarized output pulse. This second pulse is smaller than the reference value of Fig. 7 because the gain it experiences during buildup has partially been removed by the first pulse.

In the results presented so far the polymer laser emits two signal pulses, one for each polarization channel. This holds for almost all values of the interpulse delay. However, for values of this delay slightly larger than that of Fig. 8 the decaying first signal pulse is revived by the second pump pulse. A double pulse appears in the channel corresponding to the polarization of the first pump pulse followed by a single signal pulse in the other output channel. A timedomain measurement on the double pulsed output is shown in Fig. 9. The physics here is the same as that discussed in the framework of Fig. 8.

Just like the output boost of the signal in Fig. 8, the revival of the signal pulse in Fig. 9 is driven by a pump pulse of the wrong polarization. After the second pump pulse, the gain anisotropy strongly favors the nonlasing polarization mode. However, the latter contains very few photons in contrast to the lasing polarization mode; the result is highly efficient stimulated emission in the lasing polarization mode and normal (slow) buildup of the polarization mode that had been nonlasing so far. A memory effect similar to the one observed here has been observed in polarization-switching CW-driven VCSELs [33]. In that case the memory effect expresses itself as hysteresis of the lasing polarization across the polarization switch point.

An important property of our polymer gain material is that the pump-induced gain anisotropy relaxes so slowly that this relaxation can be neglected, considerably simplifying the analysis. If, instead, we would have studied a standard laser dye in a low-viscosity solvent we would not have been allowed to make such a simplifying assumption. For instance, for Coumarin 153 (a dye that photoluminesces in the same spectral region as our polymer) dissolved in methanol, the dipolar reorientation time at room temperature is $\approx 35 \mathrm{ps}$ $[11,24]$. This reorientation time is of the same order as the buildup time of the signal pulse in our laser under the prevailing pumping conditions. In that case there is appreciable relaxation of the gain anisotropy during the buildup time, but the relaxation is too slow to follow the (anisotropic) deple- tion of the gain when the signal pulse is really strong. The result is that the first signal pulse experiences reduced gain because of the reduced anisotropy, and that the excitation is less effectively depleted. This leads to the conclusion that the enhancement/suppression in the limit of short interpulse delay will be reduced. For long interpulse delay the second signal pulse can considerably profit from the remaining excitation that has become completely isotropic during the interpulse delay. Consequently, the enhancement of the second output pulse will be larger in this limit. This reasoning shows that for intermediate relaxation times of the gain anisotropy, the physical picture of the polarization cross-coupling gets more complicated. In the limit that the reorientation time is sufficiently fast that it can follow the gain depletion, the gain has become effectively isotropic at all times; in that limit the polarization properties of the laser will be mainly determined by unintended cavity anisotropies.

\section{TIME-INTEGRATED OUTPUT}

The discussion of the previous sections provides an excellent framework to understand our experimental results on the time-integrated signals in the horizontal and vertical output channels as shown in Fig. 6. For the remaining discussion we will restrict ourselves to positive values of the delay, where the horizontal pump pulse precedes its vertical partner. In that case the reference value is given by the level of the horizontal output at long delay, as in Figs. 5(a) and 7(a). Figure 6 shows the enhancement of the vertical output at long delay and that of the horizontal output at short delay. It also shows that at short delay, the vertically polarized output is suppressed. For the pump strength of Fig. 6 (normalized pump parameter $r=1.6$ ), the switch point lies at a delay of about 60 ps.

Clearly, the overall features of these time-integrated data, i.e., suppression/enhancement, the existence of a switch point where the time-integrated output in both channels are equal, will persist when the laser is driven closer to or further above threshold. This is indeed the case, as shown in Fig. 10, where the time-integrated output in both channels as a function of pump delay is plotted for values of the pump parameter equal to $r=1.2,1.6$, and 6 . For increasing pump strength one sees that the switch occurs at shorter and shorter delay, and that the difference in output level at long delays gets smaller. These experimental results are fully corroborated by the time-integrated results of our rate-equation model as presented in Fig. 11. The underlying physics is that when the pump parameter is increased the pulses get more powerful while their buildup time decreases. Consequently, the gain will deplete more fully and at an earlier time. The boundary between the regimes of short and long interpulse delays, roughly given by the end of the first emitted pulse, thus also shifts to earlier times. For large values of the pump parameter and large interpulse delay the first emitted pulse will so effectively deplete the gain that the remainder is exceedingly small. This applies to both the gain functions $K(t)$ and $M(t)$. The second pump pulse will now generate a gain that is essentially the same as that available to the first pulse [see Fig. 11(c)]. The labels $A_{1,2}$ and $B_{1,2}$ as indicated in Fig. 11(b) 


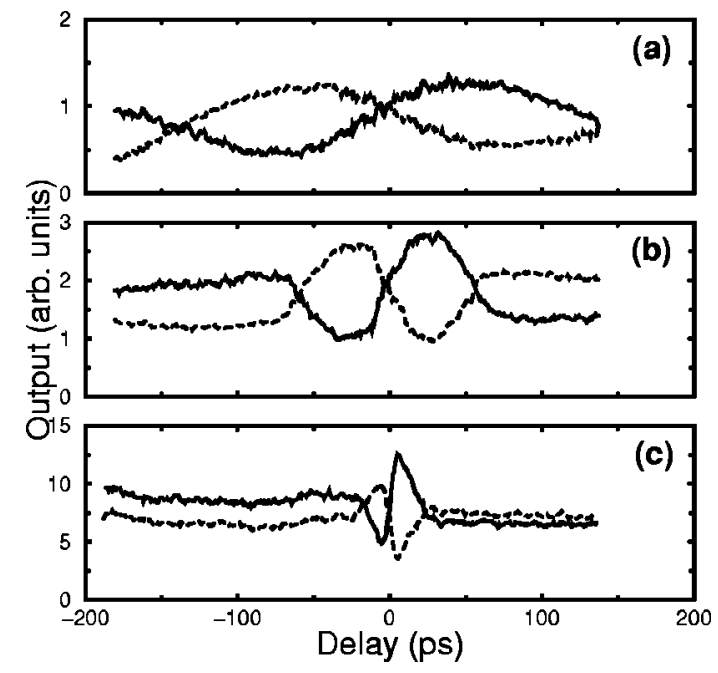

FIG. 10. Measurement of the time-integrated outputs of the horizontally (solid line) and vertically (dashed line) polarized components as a function of the interpulse delay for values of the normalized pump parameter equal to $r=1.2$ (a), $r=1.6$ (b), and $r=6(\mathrm{c})$.

correspond to the peaks having the same labels in Figs. 7 and 8 , respectively.

The switch occurs when the time-integrated outputs in both polarization channels are equal. At that value of the interpulse delay the second pump pulse excites the gain medium just when the first output pulse has extinguished. This value of the delay thus provides a convenient measure for the buildup and decay of a single pulse from such a microlaser. Therefore, the time-integrated signals allow us to get a value of a characteristic time much shorter than the response time of the measurement instruments that are used. The switch also tells us the maximum pulse repetition rate with which

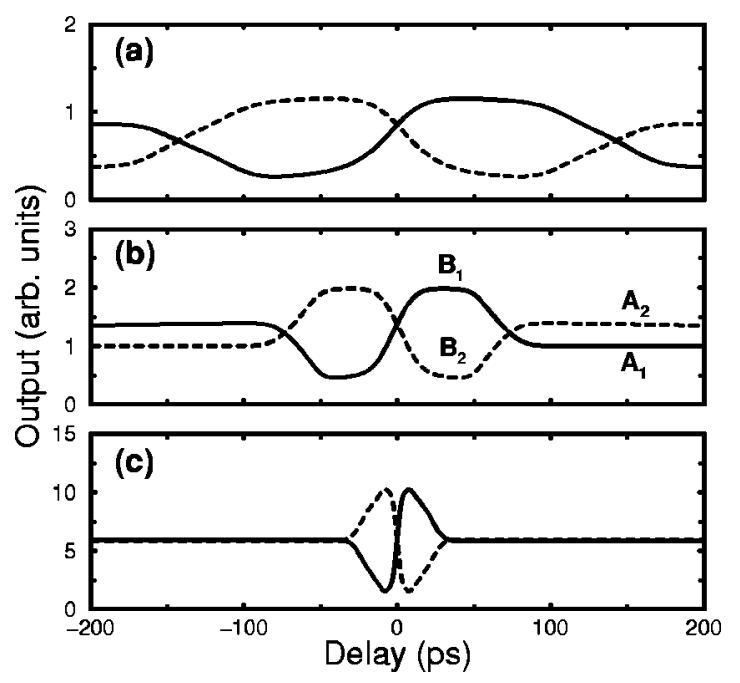

FIG. 11. Results of a numerical simulation of the horizontally (solid line) and vertically (dashed line) polarized time-integrated outputs for values of the normalized pump parameter equal to $r$ $=1.2(\mathrm{a}), r=1.6(\mathrm{~b})$, and $r=6$ (c) as a function of the delay time between the pump pulses. Positive delay corresponds to the horizontally polarized pump pulse arriving first. this laser can be driven so that it delivers well-separated output pulses, free of a CW background.

\section{CONCLUSION}

A polymer microlaser driven by two mutually delayed, orthogonally polarized, femtosecond pulses has a pulsed output in the two polarization modes defined by the polarization of the pump pulses. These two output pulses show strong cross-coupling with a remarkable dependence on the interpulse delay. The basis for this interesting behavior lies in the pump-induced gain anisotropy (the dominant anisotropy in the microlaser) and the very different time scales of our polymer microlaser. These are the duration of the pump pulse $(\approx 0.1 \mathrm{ps})$, the buildup and decay of the intracavity field (10-100 ps, depending on the pump energy), the spontaneous-emission lifetime of our fluorophore $(\approx 1 \mathrm{~ns})$, and the dipolar orientational relaxation time $(>1 \mathrm{~ns})$. An important property of the polymer is that the latter time is unusually long, considerably simplifying the physical picture.

We distinguish two regimes; for short delay the second pump pulse arrives at the moment when the signal field in the cavity, resulting from the first pump pulse, is still large. For long delay the second pulse arrives at the moment when the field resulting from the first pump pulse has become negligibly small, but some excitation may still be present in the gain medium. The existence of these regimes fully explains the observation that the dominant output polarization of the microlaser switches direction when the interpulse delay is scanned.

For small interpulse delay the field that is already present in the cavity benefits immediately from the fresh inversion that is provided by the second (orthogonally polarized) pulse. In contrast, the other polarization mode still needs to build up from the spontaneous-emission level. As a result, the latter experiences less gain and is, therefore, suppressed in this regime. For long delay, however, no field resulting from the first pump pulse is left when the inversion is boosted by the second pump pulse. In that case, the second output pulse profits from the excitation left by the first pump pulse and is, consequently, enhanced. The existence of these two regimes, a direct result of the specific order of the various time scales in the microlaser, forms the physical basis for the crosscoupling effects that we discuss in this paper.

An attractive aspect of our configuration with orthogonal pump pulses lies in the fact that the effect of the individual pump pulses on the laser output can be easily separated by measuring both polarization components separately. In this way much more information on the dynamics can be retrieved as compared to the case where two pump pulses with parallel polarizations were used.

\section{ACKNOWLEDGMENTS}

This work is part of the research program of the Stichting voor Fundamenteel Onderzoek der Materie [FOM, financially supported by the Nederlandse Organisatie voor Wetenschappelijk Onderzoek (NWO)] and Philips Research. 
[1] H. de Lang and G. Bouwhuis, Phys. Lett. 20, 383 (1966).

[2] W. van Haeringen, Phys. Rev. 158, 256 (1967).

[3] D. Lenstra, Phys. Rep. 59, 299 (1980).

[4] M.P. van Exter, M.B. Willemsen, and J.P Woerdman, Phys. Rev. A 58, 4191 (1998).

[5] M.B. Willemsen, M.P. van Exter, and J.P. Woerdman, Phys. Rev. Lett. 84, 4337 (2000).

[6] K. Otsuka, P. Mandel, S. Bielawski, D. Derozier, and P. Glorieux, Phys. Rev. A 46, 1692 (1992).

[7] A.J. Poustie, Opt. Lett. 20, 1868 (1995).

[8] G. Bouwmans, B. Ségard, and P. Glorieux, Opt. Commun. 196, 257 (2001).

[9] D.W. Phillion, D.J. Kuizenga, and A.E. Siegman, J. Chem. Phys. 61, 3828 (1974).

[10] S.K. Tiwari and S.C. Mehendale, Rev. Sci. Instrum. 69, 4245 (1998).

[11] M.L. Horng, J.A. Gardecki, and M. Maroncelli, J. Phys., Chem. A 101, 1030 (1997).

[12] T.C. Damen, L. Vi na, J.E. Cunningham, J. Shah, and L.J. Sham, Phys. Rev. Lett. 67, 3432 (1991).

[13] D. D. Awschalom and J. M. Kikkawa, Phys. Today 52(6), 33 (1999).

[14] E.L. Blansett, M.G. Raymer, G. Khitrova, H.M. Gibbs, D.K. Serkland, A.A. Allerman, and K.M. Geib, Opt. Express 9, 312 (2001).

[15] M.D. McGehee and A.J. Heeger, Adv. Mater. 12, 1655 (2000).

[16] U. Lemmer, A. Haugeneder, C. Kallinger, and J. Feldmann, in Semiconducting Polymers, edited by G. Hadziioannou and P. F. van Hutten (Wiley-VCH, Weinheim, 2000).

[17] N. Tessler, Adv. Mater. 11, 363 (1999).
[18] G. Kranzelbinder and G. Leising, Rep. Prog. Phys. 63, 729 (2000).

[19] N. Tessler, G.J. Denton, and R.H. Friend, Nature (London) 382, 695 (1996).

[20] F. Hide, M.A. Diaz-Garcia, B.J. Schwartz, M.R. Andersson, Q. Pei, and A.J. Heeger, Science 273, 1833 (1996).

[21] D. Moses, Appl. Phys. Lett. 60, 3215 (1992).

[22] H.J. Brouwer, V.V. Krasnikov, A. Hilberer, J. Wildeman, and G. Hadziioannou, Appl. Phys. Lett. 66, 3404 (1995).

[23] W. Holzer, A. Penzkofer, S.H. Gong, A.D. Davey, and W.J. Blau, Opt. Quantum Electron. 29, 713 (1997).

[24] S.A. van den Berg, G.W. 't Hooft, and E.R. Eliel, Chem. Phys. Lett. 347, 167 (2001).

[25] H. Spreitzer, H. Becker, E. Kluge, H.Schenk W. Kreuder, R. Demandt, and H.F.M. Schoo, Adv. Mater. 10, 1340 (1998).

[26] A. Costela, I. Garcia-Moreno, J. Barroso, and R. Sastre, J. Appl. Phys. 83, 650 (1998).

[27] R. Leners and G. Stéphan, Quantum Semiclassic. Opt. 7, 757 (1995).

[28] P. P. Feofilov, The Physical Basis of Polarized Emission (Consultants Bureau, New York, 1961).

[29] S.A. van den Berg, G.W. 't Hooft, and E.R. Eliel, Phys. Rev. A 63, 063809 (2001).

[30] A. Aiello, F. De Martini, and P. Mataloni, Opt. Lett. 21, 149 (1996).

[31] X. Wang, R.A. Linke, G. Devlin, and H. Yokoyama, Phys. Rev. A 47, R2488 (1993).

[32] A. E. Siegman, Lasers (University Science Books, Sausalito, CA, 1986).

[33] M.B. Willemsen, M.U.F. Khalid, M.P. van Exter, and J.P. Woerdman, Phys. Rev. Lett. 82, 4815 (1999). 